\title{
Impacto de la pandemia por COVID-19 de enero a junio de 2020 en la formación académica de residentes en la Universidad de Antioquia
}

\author{
Adolfo Alejandro López-Ríos' ${ }^{1}$, Evert Armando Jiménez-Cotes² ${ }^{(D)}$, Nelson Darío Giraldo- \\ Ramírez ${ }^{3}$ iD, Alfredo Salvador Patrón-Gómez ${ }^{4}$ (D) \\ ${ }^{1}$ Cirujano plástico, maxilofacial y de la mano. Hospital San Vicente Fundación. Medellín, Colombia. \\ ${ }^{2}$ Residente de Cirugía plástica, maxilofacial y de la mano. Universidad de Antioquia. Medellín, Colombia. \\ ${ }^{3}$ Médico anestesiólogo intensivista. Cuidado crítico cardiovascular. MSc. Epidemiologia clínica. Hospital Pablo To- \\ bón Uribe. Medellín, Colombia. \\ ${ }^{4}$ Jefe de sección cirugía plástica, maxilofacial y de la mano. Universidad de Antioquia. Medellín, Colombia.
}

\section{INFORMACIÓN ARTÍCULO}

\section{Palabras clave}

Educación;

Especialización;

Infecciones por Coronavirus;

Internado y Residencia;

Telemedicina;

Virus del SARS

Recibido: junio 2 de 2021

Aceptado: noviembre 8 de 2021

\section{Correspondencia:}

Adolfo Alejandro Lopez Ríos

aalejandro.lopez@udea.edu.co

Cómo citar: Lopez-Ríos AA, Jiménez-Cotes EA, Giraldo-Ramírez ND, Patrón-Gómez AS. Impacto de la pandemia por COVID-19 de enero a junio de 2020 en la formación académica de residentes en la Universidad de Antioquia. latreia [Internet]. 2023 Ene-Mar;36(1):98-111. https://doi.org/10.17533/udea.iatreia.164

\section{(c) (i) (5) (2)}

Copyright: @ 2023

Universidad de Antioquia.

\section{RESUMEN}

Objetivos: conocer el impacto de la pandemia del COVID-19 en la formación académica de residentes de especialidades médicas y quirúrgicas de la Universidad de Antioquia por medio de una encuesta en línea sobre la percepción de la situación durante los meses de enero a junio del 2020.

Métodos: estudio observacional, descriptivo y de corte transversal a través de una encuesta difundida entre residentes de especialidades durante el aislamiento social.

Resultados: la encuesta fue respondida por 156 residentes. El $47 \%$ convive con personas consideradas de alto riesgo para COVID-19. Todos los residentes contaron con dispositivos electrónicos y buena conectividad para realizar las actividades en línea. El 30 \% de los encuestados se ha sentido discriminado por ser personal del área de la salud. Se ha sentido satisfecho por su formación en aspectos teóricos el 81,4 \% y en actividades prácticas el 55,1\%. El riesgo de contagio por COVID-19 es el aspecto con mayor impacto negativo, reportado por el $61 \%$. Los residentes se ausentaron de sus sitios de práctica en promedio 31 días. El $52 \%$ participó en actividades de telesalud. La mayoría de los residentes (97,5 \%) participó en actividades virtuales.

Discusión: la pandemia del COVID-19 impactó significativamente los procesos formativos de los médicos residentes. La encuesta documenta una sensible disminución de las actividades asistenciales y el acceso a las actividades prácticas, con un evidente incremento de actividades por medio de las tecnologías de la información y la comunicación. La pandemia ha impulsado un proceso de adaptación en las instituciones educativas para cumplir con la planeación de las actividades programadas. 


\section{Evaluating COVID-19's impact on the training of residents at the University of Antioquia from January to June 2020}

Adolfo Alejandro López-Ríos' (iD), Evert Armando Jiménez-Cotes² ${ }^{(D)}$, Nelson Darío GiraldoRamírez ${ }^{3}$ iD, Alfredo Salvador Patrón-Gómez ${ }^{4}$ (D)

1 Plastic, maxillofacial and hand surgeon. Hospital San Vicente Fundación. Medellín, Colombia.

2 Plastic, maxillofacial and hand surgery resident. University of Antioquia. Medellín, Colombia.

${ }^{3}$ Intensive care anesthesiologist. Cardiovascular critical care. MSc. Clinical Epidemiology. Hospital Pablo Tobón Uribe. Medellín, Colombia.

${ }^{4}$ Head of Plastic, maxillofacial and hand surgery section. University of Antioquia. Medellín, Colombia.

\section{ARTICLE INFORMATION}

\section{Keywords}

Coronavirus Infections;

Education;

Internship and Residency;

SARS Virus;

Specialization;

Telemedicine

Received: June 2, 2021

Accepted: November 8, 2021

\section{Correspondence:}

Adolfo Alejandro Lopez Ríos

aalejandro.lopez@udea.edu.co

How to cite: Lopez-Ríos AA, Jiménez-Cotes EA, Giraldo-Ramírez ND, Patrón-Gómez AS. Evaluating COVID-19's impact on the training of residents at the University of Antioquia from January to June 2020. latreia [Internet]. 2023 Jan-Mar;36(1):98-111. https://doi. org/10.17533/udea.iatreia.164

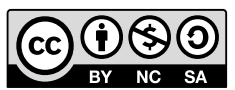

Copyright: @ 2023

Universidad de Antioquia.

\section{ABSTRACT}

Objective: To evaluate the impact of the COVID-19 pandemic on the academic development of residents in medical and surgical specialties at the University of Antioquia through an online survey.

Methods: Observational, descriptive, and cross-sectional study involving a survey distributed to medical residents.

Results: The survey was completed by 156 residents. They all had electronic devices and the means necessary to carry out virtual activities. Almost half of the participants (47.4\%) lived with people who were considered high-risk for COVID-19. Of those surveyed, 30.1\% felt discriminated against for being healthcare workers. Even though $81.4 \%$ of participants were satisfied with the theoretical training, only $55.1 \%$ were satisfied with the practical training. The most detrimental impact was the risk of contracting COVID-19, which was reported by $61 \%$ of participants. On average, residents were absent for 31 days. The $52 \%$ of residents participated in telehealth activities, and 97.5\% participated in virtual activities.

Discussion: The COVID-19 pandemic significantly impacted the training of residents. This study quantified a decrease of in-person educational activities including practical activities and an increase in virtual methods of education and communication. The pandemic has forced educational institutions to transform the way they teach in order to follow public health measures. 


\section{Introducción}

La infección por el SARS-CoV-2 (COVID-19) ha implicado cambios drásticos en los modelos de atención de pacientes. Se ha buscado optimizar los recursos y disminuir la transmisión a la comunidad en general y al personal de salud, incluidos los médicos en formación. Las acciones implementadas incluyeron cierres de fronteras, distanciamiento social, cuarentenas, cierre de instituciones académicas, suspensión del comercio y limitación de las actividades hospitalarias a solo las urgentes o emergentes (1). Estas medidas han tenido un impacto inmediato y a futuro en la formación académica de médicos y residentes.

La suspensión de cirugías electivas y procedimientos, la disminución del número de pacientes no-COVID en consulta externa, urgencias y hospitalización, además de los lineamientos de no exposición impartidos tanto por los centros educativos y sitios de práctica, han afectado al personal médico en formación tanto en Colombia como en muchos países en el mundo (2-13). La pandemia ha generado la mayor interrupción de los sistemas educativos a nivel mundial: ha afectado a 1,6 billones de estudiantes y 190 países en todos los continentes. El cierre de escuelas y centros educativos ha impactado al $94 \%$ de la población mundial estudiantil, especialmente en países de ingresos bajos y medio-bajos $(14,15)$.

Este panorama académico podría impactar la curva de aprendizaje de los residentes de especialidades médicas y quirúrgicas. Dada la ausencia de precedentes y el desconocimiento del impacto real en la adquisición de habilidades y destrezas en los residentes de especialidades médicas y quirúrgicas, este estudio pretende estimar los recursos, el tipo de adaptación y las percepciones de la población de estudiantes en formación durante el período de confinamiento inicial en el país.

\section{Métodos}

Se realizó un estudio de tipo observacional, descriptivo de corte transversal a través de una encuesta virtual en Google Forms que fue difundida entre los 417 residentes de especialidades médicas y quirúrgicas de la Facultad de Medicina de la Universidad de Antioquia, vía correos electrónicos y difusión en chats académicos.

El formulario incluyó un consentimiento informado con el propósito del trabajo, sus objetivos, posibles riesgos y datos de contacto de los investigadores. El cuestionario fue redactado por los autores luego de una revisión de la literatura. Este incluyó 65 preguntas relacionadas con aspectos sociodemográficos, técnico-informáticos, percepción personal de la pandemia por el COVID-19, actividades de formación académica asistenciales, clínicas, quirúrgicas y de telemedicina.

Los encuestados solo podían acceder al formulario desde su correo personal, lo cual permitió que cada participante contestara solo una vez. Se realizó una prueba piloto con siete residentes, la cual permitió ajustar la redacción de algunas preguntas para hacer posible una interpretación uniforme. La encuesta se distribuyó desde el 20 hasta el 27 de julio del 2020. La participación fue voluntaria y confidencial, no se recolectaron datos que permitieran identificar directamente a los participantes y se respetaron los principios bioéticos. Se incluyeron en el análisis solo las encuestas consentidas y contestadas de forma completa.

Se realizó una estadística descriptiva de porcentajes, media y desviación estándar. El proyecto fue aprobado por el Comité de Ética de la Facultad de Medicina de la Universidad de Antioquia (Acta de aprobación No. 010 de 2020). 


\section{Resultados}

De los 417 médicos residentes contactados, la encuesta fue respondida por 156 (37,41 \%). Los residentes pertenecían a las especialidades de alergología (6), anestesiología (11), cirugía de cabeza y cuello (1), cirugía general (8), cirugía pediátrica (2), cirugía plástica (11), cuidados intensivos (4), dermatología (5), endocrinología pediátrica (1), enfermedades infecciosas (1), ginecología obstetricia (10), hematología (1), medicina deportiva (2), medicina interna (6), medicina vascular (1), nefrología (1), neonatología (1), neurocirugía (5), neurología (13), neurología infantil (1), oftalmología (3), ortopedia (5), otorrinolaringología (8), patología (4), pediatría (11), psiquiatría (11), radiología (3), rehabilitación (3), toxicología (2), urgencias (14) y urología (1).

El 47,4 \% de los médicos convivían con personas consideradas de alto riesgo para COVID-19. Todos los residentes contaron con dispositivos electrónicos y buena conectividad para realizar las actividades en línea. El $30 \%$ de los encuestados se había sentido discriminado por ser personal del área de la salud. Se sintió satisfecho por la formación en aspectos teóricos el 81,4 \% de ellos, y en cuanto a las actividades prácticas, el 55,1\%.

El riesgo de contagio por COVID-19 fue el aspecto con mayor impacto negativo, reportado por el $61 \%$. El promedio de tiempo de ausencia de los sitios de práctica fue de 31 días. El $52 \%$ participó en actividades de telesalud. La mayoría de los residentes encuestados (97,5 \%) participó en actividades virtuales.

La encuesta con los respectivos resultados completos se encuentra en las Tablas 1, 2 y 3. 
Tabla 1. Encuesta y resultados $(n=156)$. Sección 1 y 2. Variables sociodemográficas y aspectos técnico-informativos

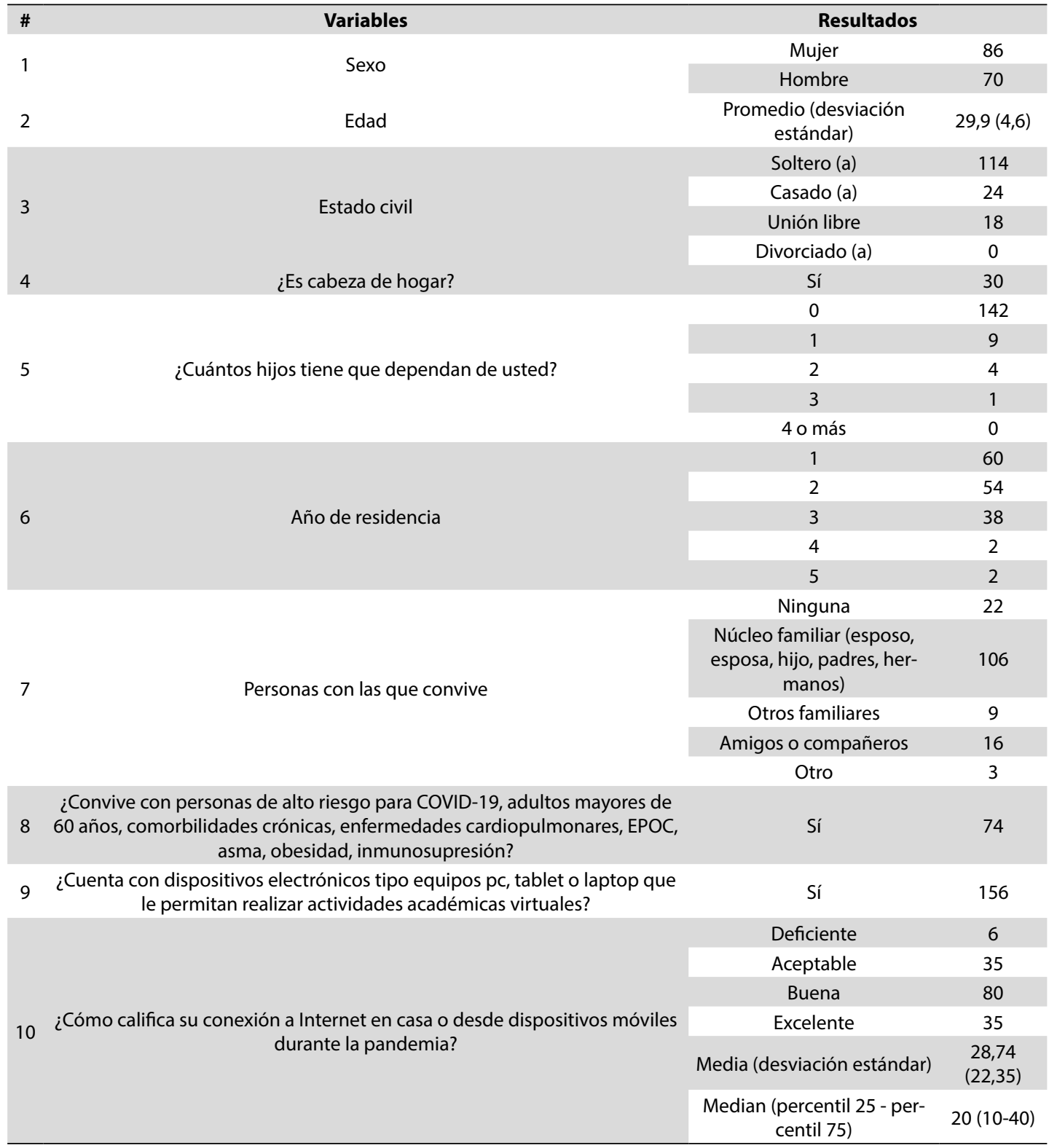

Fuente: creación propia 
Tabla 2. Encuesta y resultados $(n=156)$. Sección 3. Pandemia, percepción personal y seguridad

\# Variables Resultados

12 ¿Está de acuerdo con la declaración del estado de emergencia y activación de la cuarentena general obligatoria en Colombia?

13 ¿Se ha sentido discriminado o rechazado en ambientes no hospitalarios por ser personal de la salud?

148

Indiferente 1

Desacuerdo 7

Sí

¿Cuál es su nivel de satisfacción con respecto a la formación en aspectos teóricos recibida en su residencia durante la pandemia?

¿Cuál es su nivel de satisfacción en cuanto a formación en aspectos médico-quirúr-

15 gicos prácticos (consulta, ronda, procedimientos, cirugías) recibido en su residencia durante la pandemia?

Muy insatisfecho $\quad 30$

Insatisfecho 28

Satisfecho 97

Muy satisfecho 1

Muy insatisfecho $\quad 10$

Insatisfecho $\quad 60$

Satisfecho $\quad 76$

Muy satisfecho $\quad 10$

Deficiente 32

16

¿Cómo califica de forma global el apoyo recibido por parte de su universidad durante la pandemia?

17

¿Cuál aspecto considera ha tenido el mayor impacto negativo durante su formación académica durante la pandemia? Una o varias opciones

Aceptable 53

Bueno 52

Excelente 19

Económicas $\quad 47$

Conectividad a Internet 9

Salud-riesgo de contagio 96 por COVID-19

Movilidad 34

Familiares 43

Otro 19

18

¿Ha considerado cambiar de especialidad?

Sí 2

No $\quad 150$

Tal vez $\quad 4$

Sí 6

19 ¿Ha considerado retirarse de su programa de posgrado?

No $\quad 140$

Tal vez 10

$20 \quad$ ¿Ha tenido que mudarse de su hogar por miedo a contagiar a sus familiares?

Sí $\quad 31$

21

¿Se le ha diagnosticado infección por COVID-19 durante el ejercicio de sus prácticas académicas?

Sí

Sí

Sí

Sí

Sí

Sí

Deficiente

Aceptable

Excelente

No disponible

Poco disponible

Disponible

¿Cómo califica la disponibilidad de elementos de protección personal tipo mascarilla quirúrgica en los sitios de rotación? 
Tabla 2. Encuesta y resultados $(n=156)$. Sección 3. Pandemia, percepción personal y seguridad

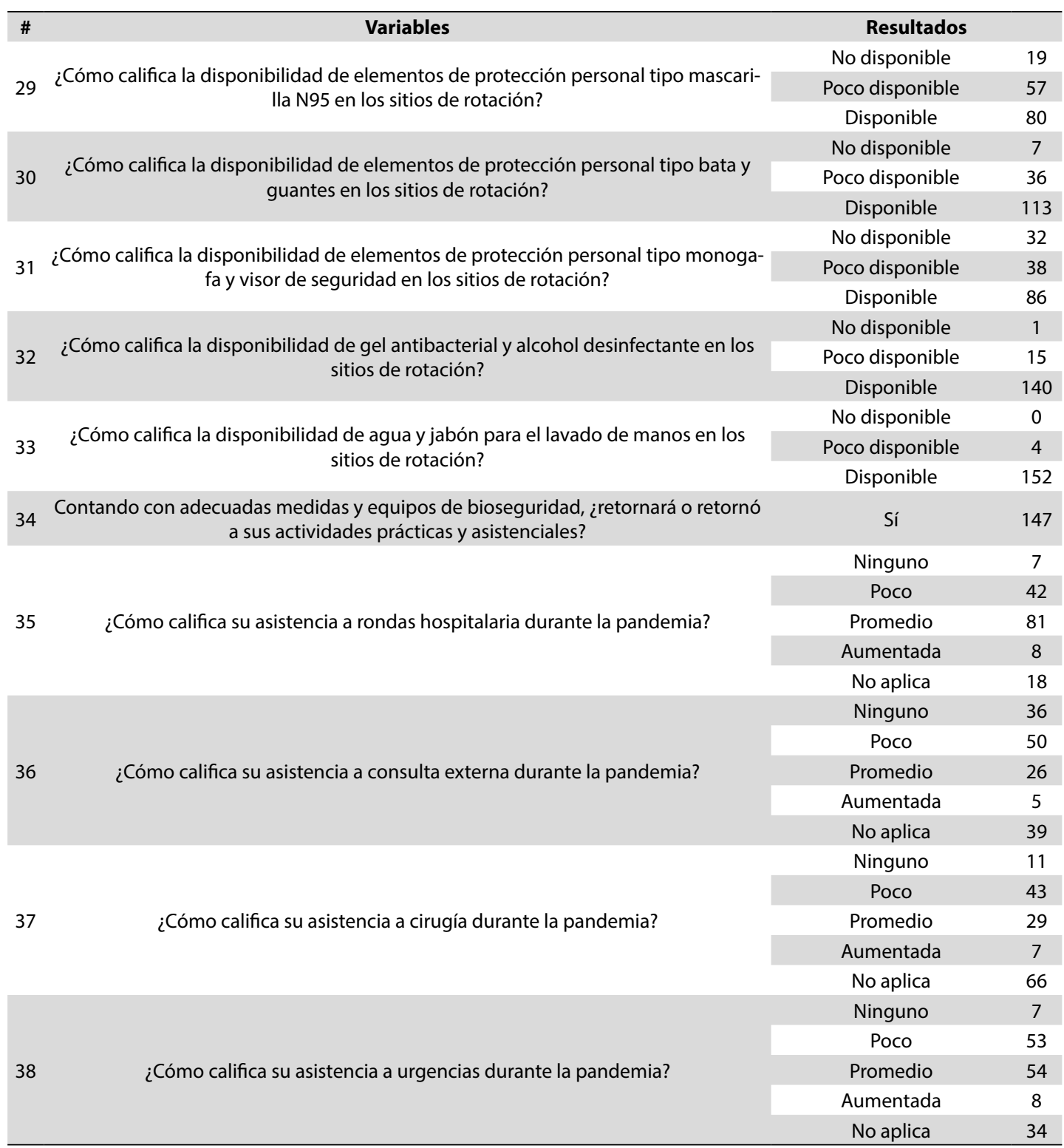

Fuente: creación propia 
Tabla 3. Encuesta y resultados $(n=156)$. Sección 4 . Actividades asistenciales, clínicas, quirúrgicas y telemedicina

\begin{tabular}{|c|c|c|c|}
\hline \# & Variables & Resultados & \\
\hline 39 & $\begin{array}{c}\text { ¿Durante la pandemia se han interrumpido sus prácticas asisten- } \\
\text { ciales en hospitales o centros de salud? }\end{array}$ & Sí & 127 \\
\hline \multirow[b]{2}{*}{40} & \multirow{2}{*}{$\begin{array}{c}\text { ¿Cuántos días ha estado o estuvo sin prácticas en los diferentes } \\
\text { sitios de rotación debido a la pandemia? (número). }\end{array}$} & Rango & $0-160$ \\
\hline & & $\begin{array}{c}\text { Median (percentil } 25 \text { y percentil } \\
75 \text { ) }\end{array}$ & $30(7-45)$ \\
\hline \multirow{6}{*}{41} & \multirow{6}{*}{$\begin{array}{l}\text { Durante la pandemia, en caso de realizar prácticas asistenciales, } \\
\text { ¿cuántos días a la semana en promedio ha asistido a consulta } \\
\text { externa? }(0,1,2,3,4,5, \text { no aplica). }\end{array}$} & 0 & 33 \\
\hline & & 1 & 17 \\
\hline & & 2 & 20 \\
\hline & & 3 & 22 \\
\hline & & 4 & 5 \\
\hline & & 5 & 7 \\
\hline \multirow{6}{*}{42} & \multirow{6}{*}{$\begin{array}{l}\text { En caso de realizar prácticas asistenciales, ¿cuántos días a la sema- } \\
\text { na en promedio ha asistido a cirugías o procedimientos? }(0,1,2,3 \text {, } \\
\qquad 4,5 \text {, no aplica). }\end{array}$} & 0 & 6 \\
\hline & & 1 & 14 \\
\hline & & 2 & 21 \\
\hline & & 3 & 6 \\
\hline & & 4 & 19 \\
\hline & & 5 & 20 \\
\hline \multirow{6}{*}{43} & \multirow{6}{*}{$\begin{array}{l}\text { En caso de realizar prácticas asistenciales, ¿cuántos días a la } \\
\text { semana en promedio ha asistido a ronda hospitalaria? }(0,1,2,3,4 \text {, } \\
\qquad 5, \text { no aplica). }\end{array}$} & 0 & 8 \\
\hline & & 1 & 7 \\
\hline & & 2 & 12 \\
\hline & & 3 & 13 \\
\hline & & 4 & 11 \\
\hline & & 5 & 80 \\
\hline \multirow{6}{*}{44} & \multirow{6}{*}{$\begin{array}{l}\text { En caso de realizar prácticas asistenciales, ¿cuántos días a la sema- } \\
\text { na en promedio ha asistido a urgencias? }(0,1,2,3,4,5, \text { no aplica). }\end{array}$} & 0 & 14 \\
\hline & & 1 & 23 \\
\hline & & 2 & 25 \\
\hline & & 3 & 13 \\
\hline & & 4 & 12 \\
\hline & & 5 & 28 \\
\hline \multirow{4}{*}{45} & \multirow{4}{*}{$\begin{array}{l}\text { ¿Cómo cuantifica según su bitácora de procedimientos el volumen de } \\
\text { pacientes quirúrgicos atendidos durante el periodo de pandemia? }\end{array}$} & Ninguno & 3 \\
\hline & & Bajo & 55 \\
\hline & & Normal o promedio & 32 \\
\hline & & Alto & 4 \\
\hline \multirow{2}{*}{46} & \multirow{2}{*}{$\begin{array}{l}\text { En el mes de enero, ¿cuántas cirugías registró en su bitácora de } \\
\text { procedimientos? si aplica (número). }\end{array}$} & Rango & $0-100$ \\
\hline & & Median (percentil 25 - percentil 75) & $25,5(9-31,5)$ \\
\hline \multirow{3}{*}{47} & \multirow{3}{*}{$\begin{array}{l}\text { En el mes de febrero, ¿cuántas cirugías registró en su bitácora de } \\
\text { procedimientos? si aplica (número). }\end{array}$} & Rango & $0-72$ \\
\hline & & Media (desviación estándar) & $19,45(15,75)$ \\
\hline & & Median (percentil 25 - percentil 75) & $20(10-30)$ \\
\hline \multirow{3}{*}{48} & \multirow{3}{*}{$\begin{array}{c}\text { En el mes de marzo, ¿cuántas cirugías registró en su bitácora de } \\
\text { procedimientos? si aplica (número). }\end{array}$} & Rango & $0-45$ \\
\hline & & Media (desviación estándar) & $12,35(13,04)$ \\
\hline & & Median (percentil 25 - percentil 75) & $8(1-21)$ \\
\hline \multirow{3}{*}{49} & \multirow{3}{*}{$\begin{array}{l}\text { En el mes de abril, ¿cuántas cirugías registró en su bitácora de } \\
\text { procedimientos? si aplica (número). }\end{array}$} & Rango & $0-41$ \\
\hline & & Media (desviación estándar) & $8,2(10,96)$ \\
\hline & & Median (percentil 25 - percentil 75) & $1,5(0-15)$ \\
\hline \multirow{3}{*}{50} & \multirow{3}{*}{$\begin{array}{l}\text { En el mes de mayo, ¿cuántas cirugías registró en su bitácora de } \\
\text { procedimientos? si aplica (número). }\end{array}$} & Rango & $0-100$ \\
\hline & & Media (desviación estándar) & $12,85(19,77)$ \\
\hline & & Median (percentil 25 - percentil 75) & $7(0-18)$ \\
\hline
\end{tabular}


Tabla 3. Encuesta y resultados $(n=156)$. Sección 4 . Actividades asistenciales, clínicas, quirúrgicas y telemedicina

\section{\# \\ 51 \\ En el mes de junio, ¿cuántas cirugías registró en su bitácora de procedimientos? si aplica (número).}

52 ¿Ha participado en actividades de telesalud durante la pandemia?

En caso de realizar actividades de telesalud, ¿cuántos días a la semana ha realizado telemedicina o teleasistencia en promedio? $(0,1,2,3,4,5$, no aplica).

En caso de estar realizando actividades asistenciales, ¿está realizando actividades académicas con los médicos y especialistas en el sitio de práctica?

¿Durante la pandemia ha tenido la oportunidad de realizar prácticas en simuladores?

¿Ha realizado actividades de investigación como elaboración de

56 proyectos, recolección de datos, análisis de información, preparación o publicación de artículos científicos durante la pandemia?

En caso de realizar actividades de investigación, ¿cuántas horas

57 a la semana ha destinado para el desarrollo de investigación? (número).

En caso de realizar actividades de investigación, ¿cuántos artículos ha sometido o preparado para publicación? (número).

\begin{tabular}{cc}
\multicolumn{2}{c}{ Resultados } \\
Rango & $0-70$ \\
\hline Media (desviación estándar) & $15,02(18,96)$ \\
\hline Median (percentil 25 - percentil 75) & $10(0-20)$
\end{tabular}

\begin{tabular}{|cc|}
\hline Sí & 82 \\
\hline 1 & 9 \\
\hline 2 & 11 \\
\hline 3 & 7 \\
\hline 4 & 29 \\
\hline 5 & 10 \\
\hline No aplica & 18 \\
\hline Ninguno & 72 \\
\hline Poco & 12 \\
\hline Normal o promedio & 40 \\
\hline Incrementado & 66 \\
\hline No aplica & 30 \\
\hline Sí & 8 \\
\hline No & 10 \\
\hline No aplica & 106 \\
\hline
\end{tabular}

(1)

Media (desvia

Sí

Rango

Media (desviación estándar)

Median (percentil 25 - percentil 75)

Median (percentil

0

1

2

3

4

5

6

Sí

Rango

114

$0-36$

$4,26(4,66)$

$4(1,5-4)$

64

60

20

4

7

0

1

59 ¿Ha participado en webinars o congresos virtuales durante el periodo de pandemia?

En caso de participar en webinars o congresos virtuales, ¿a cuán-

60 tos ha asistido entre enero a junio del 2020 aproximadamente? (número).

Media (desviación estándar) $\quad 12,67(13,69)$

Median (percentil 25 - percentil 75) $9(4-15)$

$61 \quad ¿$ ¿Ha participado en telerreuniones con docentes de su servicio durante la pandemia?

Sí

152

62 En caso de participar en telerreuniones con docentes y otros residentes ¿a cuántos ha asistido por semana en promedio? (número).

63 ¿Ha presentado seminarios académicos de forma virtual?

64 En caso de presentar seminarios académicos de forma virtual, ¿cuántos por mes ha realizado? (número).

65

Durante la pandemia, ¿cuántas horas de formación académica ha destinado por semana? (número).

\begin{tabular}{|cc|}
\hline Rango & $0-60$ \\
\hline Media (desviación estándar) & $5,21(6,94)$ \\
\hline Median (percentil 25 - percentil 75) & $4(3-5)$ \\
Sí & 156 \\
Rango & $1-40$ \\
\hline Media (desviación estándar) & $6,65(5,89)$ \\
\hline Median (percentil 25 - percentil 75) & $4(3-8)$ \\
\hline Rango & $2-112$ \\
\hline Media (desviación estándar) & $28,74(22,35)$ \\
\hline Median (percentil 25 - percentil 75) & $20(10-40)$ \\
\hline
\end{tabular}

$0-60$

Fuente: creación propia 


\section{Discusión}

La pandemia del COVID-19 ha generado un cambio drástico en los diferentes procesos. Es posible que los médicos formados bajo las circunstancias actuales tengan menos oportunidades en el desarrollo de habilidades técnicas. En el presente estudio participaron 156 residentes de la Facultad de Medicina de la Universidad de Antioquia, institución que cuenta con 46 programas de especialidades clínico-quirúrgicas, con un total de 417 residentes matriculados en el período. Los programas de especialización tienen una duración entre 3 a 5 años y los de subespecialización de 1 a 2 años (6). En la encuesta participaron residentes de todos los años de formación académica. El promedio de edad fue de 29,9 años y la mayoría eran solteros, no eran cabeza de hogar y vivían en el núcleo familiar. Este tipo de características sociodemográficas parece ser frecuente en programas de residencia en Colombia $(16,17)$.

Los residentes manifestaron haber contado con los dispositivos electrónicos suficientes para realizar las actividades académicas, al igual que conectividad a Internet. Solo el 3,8 \% consideró que su conexión fue deficiente, cifra muy baja en comparación con otros contextos de formación, especialmente en educación básica y media en países en vías de desarrollo, donde se ha visto afectada la implementación de estrategias de educación en línea por la falta de dispositivos electrónicos o conexión a Internet (4).

Desafortunadamente, 47 residentes $(30,1 \%)$ se sintieron discriminados o rechazados en ambientes no hospitalarios por ser parte del personal de la salud. Según UNICEF, el estigma social es la asociación negativa que comparte un grupo de personas al ser discriminadas o perder el estatus al conectarlas con una enfermedad (7). La estigmatización asociada al COVID-19 ha sido reportada alrededor del mundo en pacientes afectados por el virus, minorías sociales y trabajadores de la salud, especialmente asignados a atender servicios relacionados con la pandemia. Estas personas han sentido discriminación al ser rechazadas para compartir espacios comunes, además de abuso verbal, maltrato físico, chismes y devaluación social; simultáneamente, sus familiares y amigos se han sentido afectados (5,8-10).

A pesar de la pandemia, el 81,4\% de los residentes se sintieron satisfechos con el nivel de formación teórica. Por el contrario, en los aspectos prácticos como rondas, asistenciales, cirugías y procedimientos, el grado de satisfacción se redujo al 55,1 \%. Esto podría explicarse por la falta de acceso a los sitios de práctica y las restricciones asociadas, sobre todo en consultas no urgentes y cirugías electivas. Debe tenerse en cuenta que podrían existir otros factores involucrados en la percepción de satisfacción, los cuales tienen que ver con el modelo pedagógico contingente, así como la ausencia de la disponibilidad de patrones y estudios de satisfacción previos para realizar una comparación. Con relación a la percepción del soporte recibido por parte de la Universidad durante la pandemia, la mayoría (79,5 \%) lo consideró aceptable, bueno o excelente; sin embargo, el 20,5\% lo puntuó como deficiente.

De todos los participantes, 16 residentes (10,3\%) consideraron retirarse del programa. El 19,8\% tuvo que mudarse de su hogar por miedo a contagiar a sus familiares y, a la fecha de recolección de la muestra, 2 residentes (1,2\%) habían sido diagnosticados positivos para COVID-19. Se han descrito estrategias que permiten a trabajadores de primera línea vivir de forma temporal en hoteles o en sitios adaptados dentro de los hospitales con el fin de prevenir el contagio a sus contactos y cumplir con las cuarentenas implementadas en caso de ser positivos o sospechosos para la infección (10). El riesgo existente de infectarse y morir para el personal de la salud en el momento de realización de la encuesta era alto. En la mayoría de los casos se trató de médicos y enfermeros mayores de 50 años que trabajan en asilos o centros de atención en salud no hospitalarios (13).

En total, 35 residentes $(22,4 \%)$ consultaron por soporte psiquiátrico o psicológico durante este periodo, y el 53 \% manifestó que fueron afectados económicamente. No obstante, el 55,7 \% reportó 
haber realizado actividad física y deporte, y el 62,8 \% continuó con la práctica de alguna afición o actividad lúdica como ejecutar algún instrumento, manualidades o lectura de literatura no médica. El insomnio y otras alteraciones del sueño han sido documentados hasta en una tercera parte del personal de salud durante la pandemia. Estas condiciones se han relacionado con el desarrollo de actividades laborales en ambientes aislados, limitaciones de interacción con colegas, amigos, familiares y pacientes, preocupación e incertidumbre por el control de brote, y el temor de infectarse en el trabajo y trasmitir la infección a familiares y amigos (14). Las intervenciones psicosociales han demostrado ser necesarias en este tipo de crisis y sugeridas por múltiples organizaciones $(10,12,15,18)$.

La mascarilla quirúrgica, la mascarilla N95, la bata, guantes, el visor de seguridad y gel antibacterial fueron elementos identificados como poco disponibles o no disponibles por un grupo importante de los encuestados. Sin embargo, en este periodo la Universidad implementó mecanismos de distribución de elementos de protección personal para todos los residentes, con el fin de mitigar el desabastecimiento y proteger a los médicos en formación. Al comparar los datos recolectados en nuestra encuesta, encontramos que no difieren con otras en Latinoamérica, como la realizada por Delgado (19), donde la mascarilla N95 estuvo disponible en el 56,1 \% y el gel antibacterial en el $95 \%$, o la realizada por Mishra en la India (20), en la cual los elementos de protección personal fueron considerados adecuados en el $59,2 \%$.

Es de resaltar que la mayoría de los residentes (94,2\%) estaba dispuesta a retornar a sus sitios habituales de práctica con las adecuadas medidas y disponibilidad de equipos de bioseguridad. Durante los primeros seis meses de la pandemia, los residentes percibieron una gran variabilidad en la asistencia a las actividades hospitalarias como rondas, consulta externa, cirugía y urgencias. Para una tercera parte existió una disminución, con una ausencia completa en la consulta externa en el $23 \%$ de los residentes y un poco menor al $7 \%$ para la asistencia a cirugías, rondas y urgencias. Esta encuesta evidenció que el $81,4 \%$ de los residentes en este periodo interrumpió las prácticas asistenciales en hospitales o centros de salud, con una inasistencia a los sitios de práctica en promedio de 31 días. Sin embargo, la mayoría logró realizar más del $80 \%$ de las actividades programadas. Esta situación es semejante a la descrita por Zheng (21) en su estudio con residentes de último año de cirugía general, quienes, a pesar de las restricciones generadas por la pandemia, lograron obtener los requisitos mínimos para obtener su grado.

Al analizar las bitácoras de cirugías entre los residentes que realizan procedimientos quirúrgicos, es notoria la variación en la media del número de procedimientos mensuales de 23 en el mes de enero, cuando apenas iniciaba la pandemia, a 8 en abril y luego 15 para el mes de junio. Los meses de marzo, abril y mayo fueron los períodos de mayor afectación. El impacto en el entrenamiento clínicoquirúrgico de los residentes a nivel mundial ha sido percibido como deficiente, en concordancia con el estudio realizado por Mishra (20), donde el 80,7 \% percibió de manera negativa el proceso de formación, y $62,4 \%$ reportó una reducción mayor al 50 \% del entrenamiento quirúrgico.

Entre las medidas de mitigación durante la pandemia, fue notorio el incremento de actividades de telesalud, una estrategia útil y ampliamente implementada durante esta crisis (22). El presente estudio evidenció la participación de 82 residentes $(52,56 \%$ ) en actividades de telesalud, con intensidad horaria variable de acuerdo con el programa de residencia, desde un día hasta cinco días a la semana.

Múltiples publicaciones sugieren la estimulación y la implementación de prácticas en ambientes simulados con el fin de compensar los déficits generados por la falta de escenarios de práctica, además del uso de plataformas en línea con videos de cirugías pregrabadas y entrenamiento sobre procedimientos específicos que pueden ser descargados por los residentes (12,15). En el estudio, solo 10 residentes encuestados (6,41\%) tuvieron este tipo de actividades, mientras que 106 (67,65 $\%)$ no tuvieron acceso a prácticas en simuladores y 40 (25,64\%) consideraron que estas actividades no aplican para su programa formativo, a pesar de que las estrategias en simulación se pueden adaptar para cualquier tipo de entrenamiento o especialidad (23). 
Se identificó que 114 residentes (73,08\%), dedicaron parte de su tiempo a la realización de actividades de investigación. En promedio, la dedicación fue de 4 horas semanales, lo que corresponde al tiempo habitual semanal asignado por la Universidad para la investigación. Bajo esta consideración, la pandemia por COVID-19 y la falta de acceso a algunas actividades prácticas no aumentó el número de horas destinadas para investigación.

Otras actividades complementarias para mitigar los efectos de la pandemia en las actividades educativas incluyen la participación en seminarios web, telerreuniones o congresos en línea, lo que ha dado la oportunidad a estudiantes, profesores y expertos de interactuar y discutir contenido en tiempo real, con presentación de casos clínicos, con la posibilidad de tener espacios para preguntar y discutir sobre habilidades técnicas y no técnicas. Adicionalmente, los archivos pregrabados o canales de pódcast se han reportado como alternativas de aprendizaje que permiten mantener la formación en conocimientos teóricos (5,18,20,22,24-29). La mayoría de los residentes encuestados asistieron durante este periodo a eventos en línea y telerreuniones con docentes de sus programas de formación. Durante los primeros seis meses del año, en promedio, participaron en 12,65 eventos tipo seminario web o eventos académicos y en 5,21 telerreuniones en sus respectivos servicios. La totalidad de los residentes presentaron seminarios académicos en este periodo, en promedio 6,6 por mes, con un número de horas de formación académica destinada por semana en promedio de 28,64. Durante la pandemia, las reuniones y actividades de formación a través de la red fueron la norma y permitieron desarrollar y discutir aspectos teóricos.

Hay que reconocer que el presente estudio tiene varias limitantes. Primero, el sesgo de selección, donde alrededor de una tercera parte respondió la encuesta. Posiblemente la temporalidad y la naturaleza libre y voluntaria del diligenciamiento influyeron en la proporción de residentes que participaron en la misma. Segundo, las respuestas fueron autorreportadas, lo cual podría generar inexactitudes en la información, en especial al referirse a las bitácoras o registros de cirugía. De igual manera, este tipo de estudios puede no reflejar el comportamiento de la población estudiada en el contexto real. Por último, los participantes podrían suponer el propósito de las preguntas y responder ajustando sus respuestas en consecuencia (30). La tasa de respuesta de la encuesta fue del $37 \%$, por lo que no es posible generalizar los resultados a toda la población de residentes. A pesar de esto, consideramos que las respuestas obtenidas tienen un valor específico y proveen información no disponible previamente. Igualmente, con relación al universo estudiado, el tamaño de la muestra obtenida se considera óptimo, e incluso superior al reportado en otros estudios $(2,11,12,17,20)$.

Los programas de especializaciones clínicas y quirúrgicas, debido a la pandemia del COVID-19, se vieron afectados en el primer semestre del 2020 como cualquier otro proceso educativo a nivel mundial. Identificamos cómo muchos programas presentaron modificaciones en sus agendas educativas, con una disminución del número de actividades asistenciales y prácticas y un aumento significativo de las actividades basadas en la tecnología de la información y la comunicación. Los residentes reportaron haber realizado más del $80 \%$ de las actividades planeadas, adecuándose a los requerimientos mínimos necesarios. Las modificaciones pedagógicas en el contexto de la pandemia para especializaciones médicas se basan en recomendaciones para evitar afectación en la calidad educativa de los procesos de formación. El impacto y acogida de estas recomendaciones nacionales en la percepción de la formación de los residentes aún no está cuantificado. Más allá de las recomendaciones de expertos, se intenta verificar la percepción de afectación a través de un análisis basado en algunos parámetros tenidos en cuenta en la medición de la calidad en la educación de los posgrados médicos (15).

Un análisis más profundo sería necesario para estimar si existen diferencias en cuanto a la percepción de la formación, por medio de la estratificación de grupos de residentes por especialidades de manera individual en médicas y quirúrgicas. Los hallazgos del presente trabajo pueden ser un punto de partida para futuras discusiones relacionadas con la educación médica en contextos de 
limitaciones similares. Este y posteriores estudios complementarios con metodologías más robustas podrían considerarse un punto de partida para la implementación de intervenciones institucionales de bienestar estudiantil o replanteamiento de modelos pedagógicos que permitan una migración a nuevas tecnologías o el desarrollo de recursos avanzados de simulación, a la medida de competencias curriculares específicas.

\section{Conflicto de intereses}

Ninguno por declarar.

\section{Referencias}

1. Velez M, Patiño Lugo DF, Cataño D, Franco Perez JS. Síntesis Rápida: Planeación organizacional de servicios de salud para la atención de pacientes con COVID-19. Respuesta de 7 días. [Internet]. Medellín: Universidad de Antioquia; 2020. [Consultado el 17 de mayo del 2020]: [20 páginas]. Disponible en: https://acortar.link/ tkk3so

2. Albitres-Flores L, Pisfil-Farroñay YA, Guillen-Macedo K, Niño-Garcia R, Alarcon-Ruiz CA. Interns' perceptions about the medical internship suspension during the COVID-19 quarantine. Rev Peru Med Exp Salud Publica [Internet]. 2020 Dec 2;37(3):504-9. https://doi.org/10.17843/rpmesp.2020.373.5729

3. Gawad N, Towaij C, Stuleanu T, Garcia-Ochoa C, Williams LJ. Prioritizing resident and patient safety while maintaining educational value: emergency restructuring of a Canadian surgical residency program during COVID-19. Can J Surg [Internet]. 2020 May 25;63(3):E302-E305. https://doi.org/10.1503/cjs.006120

4. De Giusti A. Policy Brief: Education during COVID-19 and beyond. Rev Iberoam Tecnol En Educ y Educ En Tecnol. 2020;26:e12. https://doi.org/10.24215/18509959.26.e12

5. Costa MJ, Carvalho-Filho M. A new age for medical education after COVID-19. FEM Rev La Fund Educ Médica [Internet]. 2020;23:55-7. https://doi.org/10.33588/fem.232.1052

6. Universidad de Antioquia [Internet]. Medellín: la institución; 1996 [cited 2020 May 17]. Especialidades médicas Facultad de Medicina Universidad de Antioquia [about 1 screen]. Available from: https://bit.ly/3LSOOXO

7. Unicef. El Estigma Social Asociado con el Covid-19 [internet]. Uruguay: Unicef; 2020. [cited 2020 Nov 21]. Available from: https://uni.cf/3LKeT5N

8. Ransing R, Ramalho R, de Filippis R, Ojeahere MI, Karaliuniene R, Orsolini L, Et al. Infectious disease outbreak related stigma and discrimination during the COVID-19 pandemic: Drivers, facilitators, manifestations, and outcomes across the world. Brain Behav Immun [Internet]. 2020 Oct;89:555-8. https://doi.org/10.1016/j. bbi.2020.07.033

9. Bedoya Jojoa CM. Covid-19: La pandemia del maltrato contra el personal de la salud en tiempos de pandemia. Interdiscip J Epidemiol Public Heal. 2020;3(1):10-2. https://doi.org/10.18041/2665-427x/ijeph.1.6276

10. Abuabara YC. Ataque al personal de la salud durante la pandemia de Covid-19 en Latinoamérica. Acta med. colomb. 2020;45(3):1-15. https://doi.org/10.36104/amc.2020.1975

11. Cai Y, Jiam NT, Wai KC, Shuman EA, Roland LT, Chang JL. Otolaryngology Resident Practices and Perceptions in the Initial Phase of the U.S. COVID-19 Pandemic. Laryngoscope [Internet]. 2020 Nov;130(11):2550-7. https://doi.org/10.1002/lary.28733

12. Alhaj AK, Al-Saadi T, Mohammad F, Alabri S. Neurosurgery Residents'Perspective on COVID-19: Knowledge, Readiness, and Impact of this Pandemic. World Neurosurg [Internet]. 2020 Jul;139:e848-e858. https://doi. org/10.1016/j.wneu.2020.05.087

13. Lapolla P, Mingoli A, Lee R. Deaths from COVID-19 in healthcare workers in Italy-What can we learn? Infect Control Hosp Epidemiol [Internet]. 2021 Mar;42(3):364-365. https://doi.org/10.1017/ice.2020.241

14. Zhang C, Yang L, Liu S, Ma S, Wang Y, Cai Z, Et al. Survey of Insomnia and Related Social Psychological Factors Among Medical Staff Involved in the 2019 Novel Coronavirus Disease Outbreak. Front Psychiatry [Internet]. 2020 Apr 14;11:306. https://doi.org/10.3389/fpsyt.2020.00306 
15. Domínguez Torres LC, Torregrosa Almonacid L, Sánchez Maldonado W, Lasprilla N, Vargas Barato F, Niño Rodríguez ÁE, et al. Educación quirúrgica durante la Pandemia COVID-19: Primer consenso nacional de la División de Educación de la Asociación Colombiana de Cirugía. Rev Colomb Cirugía [Internet]. 2020;35:36372. https://doi.org/10.30944/20117582.685

16. Bermudez C, Monroy A, Torreglosa L, Henao F. Estado actual de la formación de residentes de cirugía general en Colombia. Rev Colomb Cirugía [Internet]. 2006;21:225-39. Disponible en. https://www.revistacirugia. org/index.php/cirugia/article/view/1166

17. Hernández Rodríguez P, Peña Amaya R, Bastidas Goyes A, Ríos Barbosa F. Change in the quality of life of physicians who enter in a medical- surgical specialty at a University of Colombia: a cohort study. Educ Medica [Internet]. 2021;(22)2:106-11. https://doi.org/10.1016/j.edumed.2020.09.006

18. Cabrera L, Luna C, Pedraza M. Adaptación de la residencia de Cirugía General en Colombia a la pandemia del COVID-19: programa de enseñanza quirúrgica virtual. Rev Colomb Cir [Internet]. 2020;35:257-64. https://doi.org/10.30944/20117582.632

19. Delgado D, Quintana FW, Perez G, Liprandi AS, Ponte-Negretti C, Mendoza I, et al. Personal safety during the covid-19 pandemic: Realities and perspectives of healthcare workers in latin America. Int J Environ Res Public Health [Internet]. 2020;17:1-8. https://doi.org/10.3390/ijerph17082798

20. Mishra D, Nair A, Gandhi RA, Gogate PJ, Mathur S, Bhushan P, et al. The impact of COVID-19 related lockdown on ophthalmology training programs in India - Outcomes of a survey. IJ O [Internet]. 2020;68:9991004. https://doi.org/10.4103/ijo.IJO_1067_20

21. Zheng J, Hundeyin M, He K, Sachs T, Hess DT, Whang E, Et al. General surgery chief residents' perspective on surgical education during the coronavirus disease 2019 (COVID-19) pandemic. Surgery [Internet]. 2020 Aug;168(2):222-5. https://doi.org/10.1016/j.surg.2020.06.003

22. Figueroa-Gutiérrez LM, Agredo OF, Uribe DAP, Ramírez JCD, Henao JAS, Giraldo AFM, et al. Estrategias de educación en un programa de formación en cirugía pediátrica, como respuesta a la pandemia COVID-19. Rev Colomb Cirugía [Internet]. 2020;35:553-7. https://doi.org/10.30944/20117582.780

23. George S, Cuadrado C, Solar I, Peralta J, Sanhueza H, Bascuñán J. Simulación clínica en educación médica: la experiencia del curso Síntesis de Conocimientos en Medicina. Rev Hosp Clin Univ Chile [Internet]. 2013;25:54-60. Disponible en: https://www.researchgate.net/publication/299470779_Aceptabilidad_ del_uso_de_simulacion_clinica_en_educacion_medica_la_experiencia_del_curso_Sintesis_de_Conocimientos_en_Medicina

24. Porpiglia F, Checcucci E, Amparore D, Verri P, Campi R, Claps F, et al. Slowdown of urology residents' learning curve during the COVID-19 emergency. BJU Int [Internet]. 2020;125:E15-7. https://doi.org/10.1111/ bju.15076

25. Kania K, Abu-Ghname A, Agrawal N, Maricevich RS. Four Strategies for Plastic Surgery Education amid the COVID-19 Pandemic. Plast Reconstr Surg [Internet]. 2020;146:252e-253e. https://doi.org/10.1097/ PRS.0000000000007122

26. Sánchez Ussa S, Ariza Gutiérrez A. Educación quirúrgica en Colombia en la era del COVID-19. Rev Colomb Cirugía [Internet]. 2020;35:251-6. https://doi.org/10.30944/20117582.631

27. Mohan AT, Vyas KS, Asaad M, Khajuria A. Plastic Surgery Lockdown Learning during Coronavirus Disease 2019: Are Adaptations in Education Here to Stay? Plast Reconstr Surg Glob Open [Internet]. 2020 Jul 10;8(7):e3064. https://doi.org/10.1097/GOX.0000000000003064

28. Zingaretti N, Contessi Negrini F, Tel A, Tresoldi MM, Bresadola V, Parodi PC. The Impact of COVID-19 on Plastic Surgery Residency Training. Aesthetic Plast Surg [Internet]. 2020;44:1381-5. https://doi.org/10.1007/ s00266-020-01789-w

29. Tinelli G, Sica S, Minelli F, Tshomba Y. Vascular surgery education during COVID-19 pandemic. J Vasc Surg [Internet]. 2020;72:763-4. https://doi.org/10.1016/j.jvs.2020.04.496

30. Porath $\mathrm{CL}$, Erez A. Does rudeness really matter? The effects of rudeness on task performance and helpfulness. Acad Manag J [Internet]. 2007;50:1181-97. https://doi.org/10.2307/20159919 